\title{
Factores que influyen sobre la producción científica en Contabilidad en España: la opinión de los profesores universitarios de Contabilidad (II parte)
}

\author{
Bernabé Escobar-Pérez*, Emma García-Meca**, Manuel Larrán-Jorge*** \\ *Universidad de Sevilla \\ Correo-e: bescobar@us.es \\ **Universidad Politécnica de Cartagena \\ Correo-e: emma.garcia@upct.es \\ ***Universidad de Cádiz \\ Correo-e: manuel.larran@uca.es
}

Recibido: 18-05-2013; 3a versión: 15-10-2013; Aceptado: 31-10-2013.

Cómo citar este artículo/Citation: Escobar-Pérez, B.; García-Meca, E.; Larrán-Jorge, M. (2014). Factores que influyen sobre la producción científica en Contabilidad en España: la opinión de los profesores universitarios de Contabilidad (II parte). Revista Española de Documentación Científica, 37(2):e047. doi: http://dx.doi.org/10.3989/redc.2014.2.1087

Resumen: Este trabajo es la segunda parte del publicado en la REDC, 2013, no 3, e015, siendo su objetivo analizar la opinión del profesorado universitario en Contabilidad sobre su producción científica y las causas que pueden explicar su diferencia con respecto a otras áreas afines. Para ello, realizamos una encuesta, cuyos resultados ponen de manifiesto que las causas que permiten explicar la diferencia de la producción científica en Contabilidad con otras áreas afines son muy diversas, pudiéndose destacar entre ellas la dificultad para delimitar la investigación en Contabilidad, la desmotivación por los rechazos sufridos y el esfuerzo no recompensado, así como la falta de incentivos a la investigación, el tiempo dedicado a la docencia y la falta de formación metodológica. Asimismo, hemos constatado que tanto la edad, como el tamaño de la universidad de pertenencia, la categoría profesional del profesor y la posesión de tramos de investigación influyen en su opinión sobre la producción científica contable en España.

Palabras clave: Producción científica; Contabilidad; encuestas de opinión; academia.

Factors influencing research productivity in the field of accounting in Spain: the opinions of professors of accounting ( $2^{\text {nd }}$ part $)$

Abstract: This paper is the second part of an article, the first part of which was published in REDC, 2013, n03. The aim of this paper is to analyse the opinion of academics in the field of accounting about their research productivity and the factors that could explain the differences with respect to other areas. It is a cross-sectional study of the population of members of ASEPUC by means of surveys. The results show that the causes of the differences in research productivity are explained as: problems for delimiting the research in accounting, a lack of motivation due to rejected papers and non-compensated efforts, sparse incentives for researching, and a lack of methodological training, as well as the amount of time needed for dedication to teaching activities. Tenure, professional position, productivity and university size all influence the opinion concerning scientific productivity in accounting.

Keywords: Research productivity; Accounting; opinion surveys; universities.

Copyright: () 2014 CSIC. Este es un artículo de acceso abierto distribuido bajo los términos de la licencia Creative Commons Attribution-Non Commercial (by-nc) Spain 3.0. 


\section{INTRODUCCIÓN}

Este artículo forma parte de un estudio acerca de la opinión sobre los criterios de evaluación y la producción científica en Contabilidad en España, cuya primera parte se publicó recientemente en esta misma revista (Larrán-Jorge y otros, 2013).

El análisis de las publicaciones científicas es el método más generalizado para evaluar la producción científica, proceso reconocido tan necesario como controvertido en la literatura especializada. Para los académicos, la investigación es fundamental para el acceso a puestos de mayor nivel y un mayor nivel retributivo (Mathieu y McConomy, 2003; Alexander y otros, 2007). Para las universidades es la clave para ser reconocidas como instituciones que promueven la investigación, lo que favorece su imagen y repercute en la atracción de mejores estudiantes de posgrado y mayor financiación.

Por ello, la producción científica se ha venido estudiando profusamente mediante análisis bibliométricos, con objeto de conocer la actividad científica que se ha desarrollado, entre otras, en comunidades autónomas (Alonso-Arroyo y otros, 2010), universidades (Alonso-Arroyo y otros, 2006; Maz-Machado y otros, 2010; Codina-Canet y otros, 2013), macroáreas científicas (Rojas-Sola y otros, 2008; Guardiola-Víllora y Basset-Salom, 2012; Ibáñez y otros, 2013; Pastor-Ruiz y otros, 2013), áreas de conocimiento (Bayo y Larraza, 2012; Correa Domènech y otros, 2009; Bracho-López y otros, 2012; Marcos Recio y otros, 2012), por colectivos concretos (López-Berna y otros, 2011; Torres-Salinas y otros, 2011) o sobre determinadas cuestiones de investigación (Bolaños-Pizarro, 2009; Richart-Ramón y otros, 2011). Incluso en numerosas ocasiones, sobre todo en el caso de las áreas y las cuestiones de investigación, se analizan con referencia a una revista concreta (Amat y otros, 2001; Benítez y Benavides, 2012; Santos, 2012; Ronda y Guerras, 2013).

Este proceso se institucionaliza en España a partir de 1989 con la publicación del Real Decreto $1086 / 1989$, de 28 de agosto, con el objetivo de incentivar la actividad investigadora del profesorado universitario, quedando encargada de la evaluación la Comisión Nacional Evaluadora de la Actividad Investigadora (CNEAI). Si bien son numerosos los autores que defienden que ha propiciado un aumento general de la producción científica y de su internacionalización y visibilidad (Sanz, 1995; Jiménez y otros, 2003), también lo son quienes lo ponen en cuestión en determinados aspectos (De las Heras y Polo, 2009; JiménezContreras y otros, 2011).

En esta línea, los datos de los informes que elabora con carácter periódico la CNEAI, tradicionalmente, colocan a Ciencias Sociales y Jurídicas a bastante distancia de otras como Experimentales o Ciencias de la Salud. Si atendemos a los indica- dores de producción científica por área, en 2006 Ciencias Sociales y Jurídicas fue la peor clasificada en cuanto a artículos publicados en revistas incluidas en las bases de datos del Institute for Scientific Information (ISI) y la penúltima en artículos en revistas extranjeras.

Además, al hecho de que la investigación en Ciencias Sociales es muy inferior a la de otras áreas, hay que añadir que dentro de ellas también se aprecian notables diferencias. Así, según la Fundación Española para la Ciencia y la Tecnología (2007) la investigación en Business, Management and Accounting no pasa del $4,6 \%$ del total de la investigación española, y España se sitúa en el trigésimo puesto del ranking mundial en este área, apenas un $0,7 \%$ del total mundial, mientras que en el área de Economics, Econometrics and Finance, ocupa la novena posición siendo la aportación española más del $7 \%$ de la producción de Europa Occidental. Estas dificultades de publicación fueron evidenciadas, entre otros, por Bucheit y otros (2002), quienes realizaron una comparación de los datos de las tres revistas de mayor impacto entre cuatro áreas de conocimiento de empresa para el período 1997 a 1999, mostrando que las revistas contables "top" incluían un menor número de artículos en sus números y existía una clara influencia de autores americanos (el porcentaje de autores no americanos en las revistas contables "top" era del $10 \%$, en relación al 20,3\% de Management o 15,3\% de Marketing).

En España la literatura sobre la producción científica española en Contabilidad (García y otros, 1986; Amat y otros, 1998; Amat y otros, 2001; Larrinaga, 2005; Moya y Prior, 2008) se ha basado fundamentalmente en su cuantificación, para, a partir de ella, plantear interpretaciones sobre cómo perciben los investigadores determinadas cuestiones relacionadas con la producción científica. Sin embargo, la actividad científica tiene un importante componente psicológico (percepción u opinión de la propia comunidad científica) que debe ser considerado para comprender mejor su desarrollo (Casanueva y otros, 2007).

Por ello, con la realización de este trabajo pretendemos completar la literatura previa sobre productividad científica en el área contable, teniendo en cuenta las propias opiniones de los profesores. Estas opiniones fueron obtenidas mediante una encuesta dirigida a los miembros de la Asociación Española de Profesores Universitarios de Contabilidad (ASEPUC), en la que también se les solicitaba su parecer sobre el modelo universitario actual de acreditación nacional y la valoración de los distintos criterios establecidos para la promoción universitaria (Larrán-Jorge y otros, 2013).

En consecuencia, hemos estructurado el resto de este trabajo en los siguientes apartados: en el segundo abordamos someramente los principales antecedentes que han abordado la cuestión de la producción científica contable, con especial atención 
a España. Por su parte, en el tercero presentamos los objetivos y se plantean las hipótesis a contrastar, exponiéndose la metodología en el cuarto. En el siguiente apartado, tras caracterizar la muestra desde un punto de vista académico, se exponen las opiniones de la academia contable española a nivel de área, de departamento e individual. Por su parte, en el sexto se analizan las principales diferencias observadas en los resultados obtenidos en función de los factores que permiten explicarlas. Por último, en el séptimo apartado se exponen las conclusiones alcanzadas, terminando con las referencias bibliográficas utilizadas.

\section{ANTECEDENTES SOBRE PRODUCCIÓN CIENTÍFICA EN CONTABILIDAD}

La producción en distintas áreas científicas y sus aspectos relacionados vienen siendo estudiados desde hace tiempo mediante análisis bibliométricos (Sanz, 1995), lo que demuestra su importancia, y continúan siéndolo, evidenciándose así su vigencia (Bayo y Larraza, 2012; Correa Domènech y otros, 2009; Bracho-López y otros, 2012; Marcos Recio y otros, 2012).

En el caso de la Contabilidad, la preocupación por esta cuestión comenzó en Estados Unidos en cuyo entorno se produjeron aportaciones relevantes como, entre otras, las de Zeff (1996) que analizó la calidad de las principales revistas en Contabilidad, y Lee (1997) quien estudiando los consejos editoriales de las principales revistas internacionales, concluyó que estaban dominados por una élite vinculada a determinados programas de doctorado, lo que les ha permitido controlar la academia contable. Por su parte, Panozzo (1997) investigó la situación de la investigación contable en USA y en Europa mediante la exploración de las características del período doctoral, concluyendo que el contexto europeo no es uniforme como el norteamericano descrito por Lee (1997), sino que en él convive una rica variedad de tradiciones contables nacionales que están imbuidas de los contextos en los que operan. Asimismo, Khalifa y Quattrone (2008) abordaron la situación de la producción científica en Contabilidad destacando la falta de originalidad derivada de la presión para publicar en determinadas revistas.

Con carácter general, hemos de señalar que son diversos los estudios que ponen de manifiesto la superioridad estadounidense en la producción científica contable (Lee, 1997; Panozo, 1997, Carmona y otros, 1999), atribuida fundamentalmente a la existencia de una élite, con origen en universidades de gran prestigio y con importantes barreras de entrada, frente a una comunidad contable europea con una amplia variedad en sus tradiciones contables y una menor disciplina investigadora. De acuerdo con Bucheit y otros (2002), el hecho de que la publicación esté mucho más copada por un pequeño número de instituciones académicas, especialmente en las principales re- vistas, es un rasgo típico académico de las disciplinas poco desarrolladas.

Esta preponderancia anglosajona se evidencia también a nivel europeo. Brown (1996) examinó los académicos más influyentes en el área contable, mostrando la presencia de autores británicos en los 100 artículos más citados en una media del $17 \%$. A nivel europeo el trabajo de Carmona y otros (1999) analiza los artículos publicados en trece revistas "top" durante el periodo 1992-1997, evidenciando también la importancia de los autores británicos, que publicaron $2 / 3$ de los trabajos en las revistas analizadas a pesar de representar sólo el $20 \%$ de los miembros de la European Accounting Association. La facilidad de utilizar su mismo idioma para escribir, la temprana orientación británica hacia la investigación académica y la mayor tradición en la valoración de las publicaciones de sus investigadores en revistas de calidad para la promoción justificarían estas diferencias. Sus resultados también pusieron de manifiesto la mayor productividad en revistas "top" de los académicos que investigaban cuestiones relativas a Contabilidad Financiera, en comparación con aquellos dedicados a Contabilidad de Gestión. De forma similar Jones y Roberts (2005) abordaron la productividad de académicos en revistas de calidad mostrando que el $87 \%$ de los artículos publicados en 12 revistas "top" durante el periodo 1996-2000 proviene de USA, Reino Unido, Australia, Canadá y Hong-Kong. También Raffournier y Schatt (2009) evidenciaron que la mayoría de las revistas contables "top" publicaban trabajos de autores residentes en países de habla inglesa y relativos a temas financieros, poniendo de manifiesto que el idioma y la línea de investigación son factores fundamentales a la hora de justificar las diferencias en productividad.

Asimismo, la productividad científica también varía dentro de un mismo país en función de aspectos tales como la calidad de sus programas de doctorado y el prestigio de las facultades, siendo estos aspectos evidenciados empíricamente por trabajos realizados en USA (Maranto y Streuly, 1994; Fogarty y Ruhl, 1997; Fogarty, 2004).

En el caso de España, entre los trabajos que analizan la evolución de la investigación en Contabilidad, cabe destacar los estudios de García y otros (1986), Cañibano y Gonzalo (1997), Amat y otros (1998), Larrinaga (2005), Moya y Prior (2008) y Argilés y García (2011). A estos trabajos hay que añadir los estudios que examinan la evolución de áreas concretas dentro de la Contabilidad, entre los que podemos citar los de Donoso (2005), Escobar y otros (2005), Hernández (2005), Brusca (2010) y Lunkes y otros (2011). Para el caso de la Contabilidad Pública, Brusca (2010) analizó la evolución de la investigación en los últimos treinta años, poniendo de manifiesto una notable internacionalización de los resultados de los trabajos, no sólo mediante la presentación de los mismos en workshops y conferencias internacionales, sino 
también con la publicación en revistas internacionales con índice de impacto, especialmente en el último quinquenio. Esta evolución temporal hacia la expansión de los resultados de los trabajos de Contabilidad a las revistas internacionales refleja adecuadamente la situación existente en general dentro de la investigación en Contabilidad.

En los estudios más recientes sobre productividad científica contable en España se hace referencia a los factores determinantes de la misma. Uno de los trabajos pioneros es el de Larrinaga (2005), quien avanzando en la línea de los estudios descriptivos de la producción científica de los investigadores españoles (García y otros, 1986; Amat y otros, 1998 y 2001) y europeos (Carmona y otros, 1999), abordó algunos factores asociados a la publicación de artículos académicos. Sus hallazgos le permitieron concluir que el factor institucional es el que parece estar más relacionado con el nivel de producción científica, ya que algunos departamentos universitarios tenían una mayor producción científica que otros, mientras que los incentivos profesionales no eran demasiado determinantes. Estos resultados fueron avalados posteriormente por Moya y Prior (2008), quienes evidenciaron que la mayor parte de la producción científica en Contabilidad se localizaba en departamentos históricamente muy activos, así como la clara orientación de las nuevas universidades hacia la actividad investigadora.

Por su parte, Casanueva y otros (2007) aplicaron el análisis de redes sociales al estudio de la comunidad científica contable en España, identificando una estructura social en cuyo núcleo aparece un grupo de 22 catedráticos con una dilatada experiencia, especialmente activos en la producción de tesis doctorales y en la presencia en los tribunales que las juzgan. Sin embargo, resulta curioso que entre quienes ocupan posiciones centrales y periféricas en la red, no existen diferencias significativas en cuanto a su producción científica. Asimismo, Casanueva y Caro (2013), en una investigación posterior, muestran que los factores sociales influyen positivamente en una actividad académica y científica de forma tan relevante como la obtención del grado de doctor, mientras que la productividad científica individual no lo hace.

Por último, también Argilés (2009) aporta elementos objetivos de juicio mediante la comparación con otras disciplinas académicas, reflexionando sobre las posibilidades de llevar a cabo con éxito una carrera académica en Contabilidad, destacando unos vicios de funcionamiento existentes en el propio interior del área, entre los cuales juegan un papel importante las oportunidades de mercado, la poca tradición de investigación, la separación entre la práctica profesional de la Contabilidad y la investigación, la escasa formación investigadora de los académicos contables y la tradicional desatención de la investigación como objetivo por parte de los académicos. A su vez, según se desprende del re- ciente estudio de Cavero y Fernández (2013), en la valoración de la investigación contable influye negativamente la escasa presencia que hasta el momento los profesores de dicha subárea han tenido en las comisiones de evaluación y que esta mínima representación conlleva unos peores resultados en la evaluación de los profesores del subárea contable.

\section{OBJETIVO E HIPÓTESIS}

La mayor parte de los estudios de productividad emplean como unidad de análisis los autores individualmente $\mathrm{y} / \mathrm{o}$ agrupados por los departamentos, centros y/o universidades a los que están adscritos o incluso los programas de doctorado de los que provienen, para ofrecer mediante el recuento de artículos o el análisis de citas, una visión sobre la productividad científica en una determinada área de investigación, periodo, o entorno geográfico (Amat y otros, 1998 y 2001; Moya y Prior, 2008).

A diferencia de ellos, en este trabajo se aborda un doble objetivo. Por un lado, conocer la opinión del profesorado universitario en Contabilidad sobre las causas que podrían explicar la diferencia entre su producción científica con respecto a otras afines. Por otro, hemos analizado si existen diferencias de opinión en función de la edad, la universidad a la que pertenece, la posesión de tramos de investigación o la categoría profesional.

Como pretendemos no sólo aportar datos descriptivos, sino examinar las causas que pueden explican las diferencias de opinión sobre la productividad, para establecer las hipótesis del estudio nos hemos basado en la literatura previa que hace referencia no sólo a los métodos de evaluación, sino también a los diferentes factores explicativos de la mayor o menor productividad, tanto institucionales como individuales que han sido analizados a nivel general (Dundar y Lewis, 1998; García, 2007) o en determinadas áreas de conocimiento (Kotrlik y otros, 2002; Bland y otros, 2005).

De acuerdo con Larrinaga (2005), los departamentos de pertenencia influyen sobre la producción y la productividad científica, debido al entorno académico en el que se desenvuelven los profesores o a los incentivos que los departamentos proporcionan, en términos económicos, de oportunidades profesionales, etc. En este sentido, el trabajo de Fogarty (2004) sobre una muestra de profesores senior americanos de Contabilidad, obtuvo como indicador fundamental de su productividad el prestigio de la institución a la que pertenecía. También Maranto y Streuly (1994) obtuvieron resultados similares para los primeros años de carrera del profesor universitario en Contabilidad en USA, poniendo de manifiesto la influencia de aspectos como la calidad de los programas de doctorado o la obtención de financiación externa. Resultados similares fueron obtenidos por Fogarty y Ruhl (1997).

En consecuencia, consideramos que el tamaño de la universidad, como subrogado de su tradición 
y recursos, podría ser un condicionante de su opinión sobre la productividad en España. Atendiendo a lo anterior, la primera hipótesis a contrastar es:

H1: Existen diferencias en la opinión sobre la productividad del profesor universitario en Contabilidad en función del tamaño de la universidad a la que pertenece.

Por otro lado, la edad del profesor podría explicar las diferencias en su opinión sobre las diferencias en la productividad contable respecto a otras áreas afines. La literatura previa ha demostrado en este sentido una fuerte influencia de la edad sobre la productividad científica (Bayer y Dutton, 1977; Bentley y Blackburn, 1990). Así pues, la hipótesis a contrastar es la siguiente:

H2: Existen diferencias en la opinión sobre la productividad del profesor universitario en Contabilidad según la edad del mismo.

Asimismo, la evidencia empírica ha demostrado una fuerte asociación entre el rango académico del profesor universitario y su nivel de productividad (Astin y Davis, 1985; Bellas y Toutkoushian, 1999). Este factor estaría ligado a incentivos profesionales y académicos que tienen o perciben los investigadores en Contabilidad, esperando que la producción científica del cuerpo de mayor rango fuera sustancialmente mayor que la de los demás (Larrinaga, 2005). Por tanto, la hipótesis a contrastar es:

H3: Existen diferencias la opinión sobre la productividad del profesor universitario en Contabilidad en relación a la categoría profesional a la que pertenece.

Finalmente, hemos analizado si existe una asociación entre la opinión sobre la productividad y la propia productividad del encuestado. Para ello, nos basamos en un indicador de productividad medido de manera formal como es la concesión de tramos de investigación, distinguiendo entre aquellos pro- fesores que cuentan al menos con un sexenio y aquellos que no. En función de ello, la hipótesis a contrastar es la siguiente:

H4: Existen diferencias en la opinión sobre la productividad del profesor universitario en Contabilidad según la posesión o no de tramos de investigación.

\section{METODOLOGÍA}

Los datos de este trabajo proceden de las opiniones sobre la producción científica en Contabilidad, obtenidas mediante una encuesta que fue dirigida a 910 miembros de ASEPUC, cuyas características se pueden consultar en Larrán-Jorge y otros (2013).

Para un mayor detalle, en primer lugar, se clasificó la muestra según número de matriculados y graduados de la universidad a la que pertenece cada encuestado, en conglomerados según sus k-medias, obteniéndose 3 grupos claramente diferenciados: universidades pequeñas $(35,53 \%)$, grandes $(28,43 \%)$ y muy grandes $(36 \%)$, estando representadas la mayor parte de las universidades públicas españolas (Larrán-Jorge y otros, 2013).

En segundo lugar, en la muestra se distinguieron tres grandes grupos de edad: menor o igual a 30 años $(3,41 \%)$, entre 31 y 45 años $(61,95 \%)$ y, finalmente, mayor o igual a 46 años $(34,63 \%)$.

En tercer lugar, tomando como base las categorías profesionales se clasificó la muestra en los siguientes grupos (Figura 1): Funcionario Doctor $(46,83 \%)$, Funcionario No Doctor $(15,61 \%)$, Laboral Doctor $(13,17 \%)$, Laboral No Doctor $(12,20 \%)$ y Otros $(12,20 \%)$. Sobre este particular podemos señalar que la muestra posee una alta representatividad al compararla con los datos sobre la composición del profesorado universitario de Contabilidad obtenidos por Illueca y García (2009) de las páginas web de las universidades ${ }^{1}$.

Figura 1. Encuestados según grupos profesionales

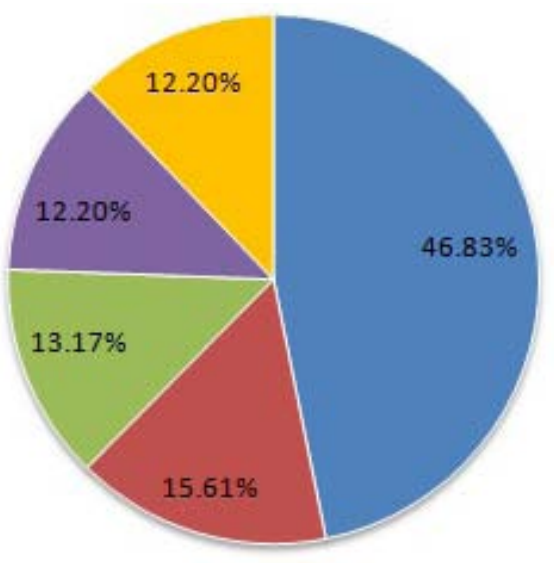

- Funcionario Doctor: $46,83 \%$

a Funcionario No Doctor: $15,61 \%$

= Laboral Doctor: $13,17 \%$

Laboral No Doctor: $12,20 \%$

Otros: $12,20 \%$ 
Por último, en relación a los sexenios, el 29,38\% de la muestra ha solicitado en algún momento de su carrera profesional un tramo de investigación (en concreto, 62 profesores), mientras que más del $70 \%$ nunca lo ha solicitado (Figura 2). De los 116 sexenios solicitados, 83 fueron concedidos, lo que supone un $71,55 \%$. En términos unitarios medios tampoco existe demasiada diferencia entre el número de sexenios solicitados $(1,87)$ y concedidos $(1,69)$, siendo el número de sexenios concedidos para Catedráticos de Universidad de 2,56 en término medio y de 1,19 para Titulares de Universidad.

Como también puede observarse en la Figura 2 , el $6,16 \%$ del total de encuestados solicitaron sin éxito un tramo de investigación, alrededor del $14,22 \%$ posee un tramo, $4,74 \%$ dos tramos y sólo el $4,26 \%$ tres o más. Esto pone de manifiesto que la situación actual de la productividad científica en los profesores del área contable, aunque haya mejorado en los últimos años, dista aún del nivel esperado según el número de investigadores de las universidades españolas. Por su parte, aunque las diferencias no son estadísticamente significativas, se constata que el número medio de tramos concedidos es de 1,53 en universidades pequeñas, 2,15 en grandes y 1,29 en universidades muy grandes.

\section{RESULTADOS DE OPINIÓN SOBRE LA PRO- DUCCIÓN CIENTÍFICA EN CONTABILIDAD}

Antes de exponer las opiniones de los encuestados sobre la producción científica en Contabilidad, comentamos su perfil investigador, debido a que éste nos ayudará a comprender mejor los resultados obtenidos. Para determinarlo se les preguntó sobre las siguientes cuestiones: No de años necesarios para finalizar tesis doctoral, Movilidad, Años transcurridos para obtención de plazas, Dedicación a tareas de docencia, investigación y gestión, Dirección de tesis doctorales y proyectos de investigación, Participación en congresos, Estancias pre y post-doctorales, Tramos de investigación solicitados/concedidos, Líneas de investigación. Con ello podemos analizar la dedicación del profesorado a las distintas tareas: docentes, investigadoras y de gestión, así como su caracterización según su categoría profesional, tramos de investigación, edad y universidad.

En cuanto a la dedicación, se pone de manifiesto que el $93 \%$ desarrolla su actividad universitaria a tiempo completo, dedicando una media de 14,37 horas semanales a la docencia (35\%), 18,58 a la investigación (45\%) y 8,24 a la gestión universitaria $(20 \%)$. Los resultados contrastan con los obtenidos por García y otros (1995) para el año 1994, en el que el profesorado universitario dedicaba su tiempo preferentemente a tareas docentes $(60 \%$ del tiempo frente al $27 \%$ dedicado a investigación). Al dividir la muestra en grupos de acuerdo con la edad (Tabla I), observamos diferencias estadísticamente significativas en relación a las horas semanales dedicadas a la docencia, la investigación y la gestión. En concreto, mientras que el grupo de edad menor o igual a 30 años se dedicada más a la investigación, el profesorado mayor o igual a 46 años ocupa más tiempo a las tareas de gestión.

Figura 2. Porcentajes de profesorado según tramos de investigación

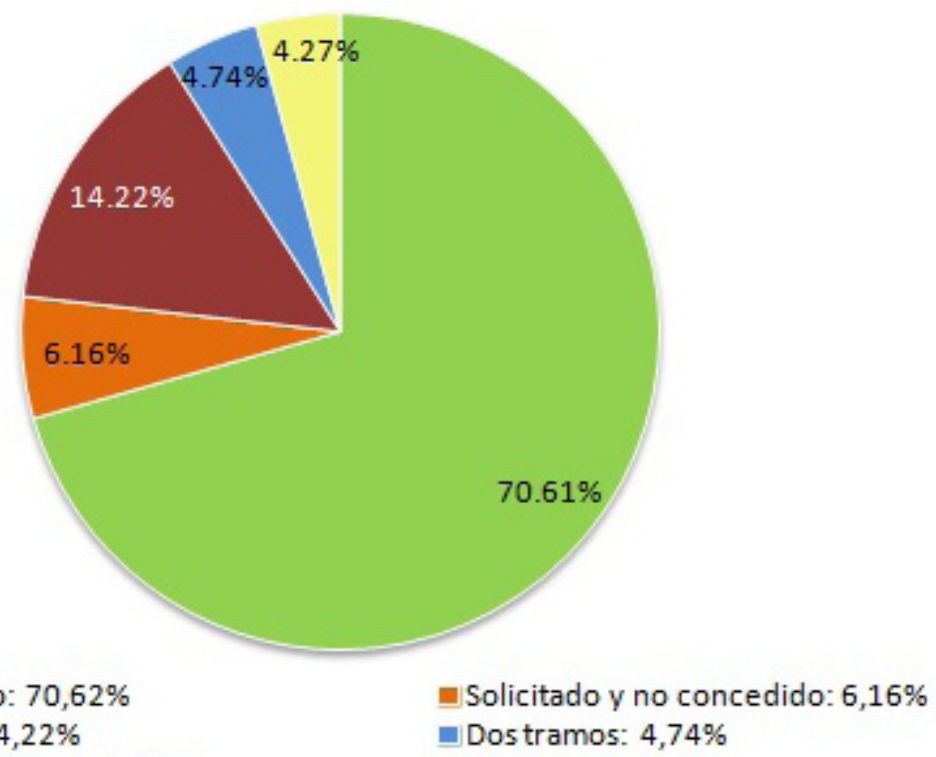

No solicitado: $70,62 \%$

Dos tramos: 4,74\%

Tres o más tramos: $4,27 \%$ 
Tabla I. Tiempo dedicado a tareas y factores explicativos

\begin{tabular}{|c|c|c|c|}
\hline Diferencias Perfil Académico & & Media & \\
\hline Tiempo dedicado a tareas & Docencia & Investigación & Gestión \\
\hline \multicolumn{4}{|l|}{ Edad } \\
\hline Menos de 30 años & 13,71 & 29,29 & 4 \\
\hline Entre 30 y 45 años & 15,60 & 18,23 & 5 \\
\hline Más de 45 años & 12,13 & 18 & 10,03 \\
\hline Chi-2 & 9,152 & 7,817 & 6,965 \\
\hline Signif. & 0,010 & 0,020 & 0,031 \\
\hline Tamaño Universidad & Docencia & Investigación & Gestión \\
\hline Pequeña & 14,39 & 19,40 & 9,76 \\
\hline Grande & 12,60 & 18,90 & 7,33 \\
\hline Muy grande & 15,78 & 17,60 & 7,67 \\
\hline Chi-2 & 6,822 & 0,288 & 3,129 \\
\hline Signif. & 0,023 & 0,866 & 0,209 \\
\hline Categoría & Docencia & Investigación & Gestión \\
\hline Funcionario doctor & 13,03 & 18,31 & 10,67 \\
\hline Funcionario no doctor & 17,45 & 18,33 & 5,93 \\
\hline Laboral doctor & 13,96 & 23,48 & 5,5 \\
\hline Laboral no doctor & 16,13 & 19,21 & 6,04 \\
\hline Otros & 13,73 & 12,67 & 6,64 \\
\hline Chi-2 & 7,681 & 12,455 & 17,357 \\
\hline Signif. & 0,104 & 0,014 & 0,002 \\
\hline Tramos Investigación & Docencia & Investigación & Gestión \\
\hline $\mathrm{Si}$ & 12,41 & 20,79 & 10,14 \\
\hline No & 15,31 & 17,55 & 7,33 \\
\hline U-Mann Withey & 3013,5 & 2914 & 2885 \\
\hline Signif. & 0,015 & 0,018 & 0,014 \\
\hline
\end{tabular}

Un factor fuertemente discriminante respecto a las horas de docencia es el tamaño de la universidad, siendo mayor el tiempo dedicado a estas tareas en las universidades muy grandes. Respecto a las categorías de profesorado las diferencias son estadísticamente significativas en las horas dedicadas a investigación, mayores para el profesorado Laboral Doctor; y a la gestión, donde los Funcionarios Doctores se dedican más que el resto. Por su parte, en cuanto a los tramos de investigación, los profesores que cuentan con algún tramo dedican menos horas a la docencia y más a actividades de investigación $y$, curiosamente, a la gestión, siendo las diferencias significativas para las tres actividades analizadas.
En la Tabla II puede apreciarse que la mayoría de los que respondieron había participado en algún proyecto de investigación obtenido en convocatoria pública. Bastante mayor es la participación en congresos, tanto en España, donde destacan los Funcionarios Doctores, como en el extranjero en los que priman los Laborales Doctores, a pesar de que los Funcionarios No Doctores no llegan ni a la mitad de los anteriores. Por el contrario, resulta llamativo el escaso porcentaje de la muestra, $28 \%$, que ha realizado estancias predoctorales, destacando en este apartado los Laborales Doctores y No Doctores.

Tabla II. Perfil académico por categoría profesional

\begin{tabular}{lcccccccc}
\hline & Media & FD & FND & LD & LND & Otros & Chi-2 & Sig. \\
\hline Participación proyectos & 0,73 & 0,80 & 0,47 & 0,96 & 0,88 & 0,50 & 31,089 & $\mathbf{0 , 0 0 0}$ \\
\hline Contrib. Congresos Nacionales & 4,62 & 5,10 & 3,77 & 4,96 & 4,59 & 3,38 & 11,126 & $\mathbf{0 , 0 2 5}$ \\
\hline Contrib. Congresos Internac. & 4,23 & 4,60 & 1,83 & 4,88 & 0,56 & 0,08 & 23,075 & $\mathbf{0 , 0 0 0}$ \\
\hline Estancias Predoctorales & 0,28 & 0,27 & 0,09 & 0,50 & 0,56 & 0,08 & 26,262 & $\mathbf{0 , 0 0 0}$ \\
\hline
\end{tabular}


Por otra parte, en la Tabla III en relación a los tramos de investigación, también se observa que las estancias, en este caso postdoctorales, siguen siendo escasas, si bien en este apartado los profesores con sexenio doblan a los que no lo tienen. Asimismo, el profesorado con algún sexenio participa más en proyectos de investigación, siendo mayoría aplastante en cuanto al número de veces en que actúa como investigador principal (65\% con sexenio vs. $20 \%$ sin él), quedando la media reducida a tan sólo el 33\%.

Como se observa en la Tabla IV, otro factor discriminante respecto a la movilidad internacional es la edad, destacando ampliamente el profesorado menor o igual a 30 años en cuanto a las estancias predoctorales. Esto pone de manifiesto la cada vez mayor presión hacia una proyección internacional en el período formativo y la creciente adecuación del profesorado español a las exigencias de movilidad internacional. Las diferencias son también significativas según el tamaño de las universida- des. Así, aunque las universidades muy grandes destacan porque sus miembros realizan un mayor número de estancias predoctorales, su posición pasa al último lugar si analizamos el número de estancias postdoctorales.

A continuación se exponen los resultados obtenidos acerca de las opiniones de los encuestados sobre la diferencia de productividad entre la Contabilidad y otras áreas, a nivel global, departamental e individual.

A nivel global del Área (Tabla V), entre las causas señaladas en las respuestas obtenidas destacan la desigualdad de criterios con respecto a otras áreas, en especial, las Jurídicas; la desmotivación por los rechazos sufridos, tanto en sexenios como en proyectos y acreditaciones. La fiabilidad y validez de la encuesta se calculó a través del coeficiente de Cronbach, obteniendo tras su análisis un índice alpha de 0,664, lo que indica un nivel aceptable de consistencia interna.

Tabla III. Perfil académico por tramos investigación

\begin{tabular}{lccccc}
\hline & Media & No sexenio & Sexenio & U-MW & Sig. \\
\hline Dirección tesis & 0,35 & 0,20 & 0,71 & 2271 & $\mathbf{0 , 0 0 0}$ \\
\hline Participación proyectos investigación & 0,73 & 0,66 & 0,89 & 3359 & $\mathbf{0 , 0 0 1}$ \\
\hline Dirección proyectos investigación & 0,33 & 0,20 & 0,65 & 2169 & $\mathbf{0 , 0 0 0}$ \\
\hline Estancias postdoctorales & 0,12 & 0,09 & 0,19 & 4128 & $\mathbf{0 , 0 0 3}$ \\
\hline
\end{tabular}

Tabla IV. Perfil académico por edad y universidad

\begin{tabular}{lccc}
\hline Estancias predoctorales & Media \% & Chi-2 & Signif \\
\hline Edad & & & $\mathbf{0 , 0 0 0}$ \\
\hline Menos de 30 años & $86 \%$ & 27,911 & \\
\hline Entre 30 y 45 años & $35 \%$ & & \\
\hline Más de 45 años & $9 \%$ & & \\
\hline Tamaño Universidad & & & \\
\hline Pequeña & 1,37 & & Signif \\
\hline Grande & 1,45 & & \\
\hline Muy grande & 2,25 & & $\mathbf{0 , 0 4 5}$ \\
\hline Estancias postdoctorales & Media $\mathbf{n}^{\mathbf{2}}$ & & \\
\hline Tamaño Universidad & & & \\
\hline Pequeña & 0,42 & & \\
\hline Grande & 0,44 & & \\
\hline Muy grande & 0,33 & & \\
\hline
\end{tabular}

Tabla V. Opiniones sobre la productividad a nivel de área

\begin{tabular}{lcc}
\hline Causa: & Media & DT \\
\hline Desigualdad de criterios respecto a otras áreas, tales como las jurídicas & 4,10 & 1,29 \\
\hline Desmotivación por rechazos (proyectos, sexenios, acreditaciones) & 3,91 & 1,25 \\
\hline $\begin{array}{l}\text { Dificultad en diferenciar la investigación, en algunos casos, de trabajos más relacionados con la } \\
\text { mejora de la práctica profesional }\end{array}$ & 3,60 & 1,22 \\
\hline Falta de formación en metodología de investigación & 3,59 & 1,24 \\
\hline Escasez de revistas específicas de calidad en Contabilidad & 3,49 & 1,30 \\
\hline Escasa tradición investigadora & 3,42 & 1,31 \\
\hline Escasos cursos de doctorado de calidad & 3,22 & 1,32 \\
\hline Falta de identidad del área & 3,04 & 1,32 \\
\hline Dificultades idiomáticas & 3,03 & 1,24 \\
\hline
\end{tabular}


Por otra parte, entre las causas que justifican la diferencia de productividad en Contabilidad a nivel de departamento (Tabla VI), sobresalen la desmotivación por los rechazos sufridos y la presencia de actitudes acomodadas. Con menor importancia encontramos la falta de incentivos a la investigación y la falta de reconocimiento de la actividad investigadora al asignar la docencia. Con relación a esta última cuestión, hemos de resaltar que ya algunas universidades públicas españolas, valoran las actividades de investigación, gestión y docencia del profesor universitario, permitiendo una reducción de créditos que puede llegar incluso hasta el 50\% de su carga docente total y en las que se valoran aspectos como la posesión de tramos de investigación, la participación en proyectos, la pertenencia a comités editoriales o la dirección de tesis doctorales. El coeficiente alpha de Cronbach muestra un valor de 0,796 , lo que indica un nivel satisfactorio de consistencia interna.

Con respecto a la opinión de los encuestados sobre la diferencia de productividad en Contabilidad a nivel individual (Tabla VII), resaltan el tiempo dedicado a la docencia, el esfuerzo no recompensado y la falta de formación metodológica, siendo el alpha de Cronbach de 0,815. Sobre la formación metodológica debemos señalar que durante los últimos años se ha observado un cambio importante en los programas de doctorado de las universidades públicas españolas en el ámbito de Contabilidad, las cuales incorporan cada vez más una mayor formación estadística que facilita la elaboración de trabajos de investigación empíricos. Por otro lado, la causa que menos influye según los encuestados es el tiempo dedicado al ejercicio profesional.

Tabla VI. Opiniones sobre la productividad por departamento

\begin{tabular}{|c|c|c|}
\hline Causa: & Media & DT \\
\hline Desmotivación por rechazos (proyectos, sexenios, acreditaciones) & 3,86 & 1,30 \\
\hline Presencia de actitudes acomodadas & 3,75 & 1,25 \\
\hline Falta de incentivos a la investigación (financiación) & 3,57 & 1,26 \\
\hline Falta de reconocimiento de la investigación en la asignación de docencia & 3,51 & 1,34 \\
\hline Falta de formación en metodología de investigación & 3,41 & 1,37 \\
\hline Escasez de revistas específicas de calidad en Contabilidad & 3,37 & 1,33 \\
\hline Dificultades para la formación de grupos de investigación & 3,25 & 1,37 \\
\hline Escasa tradición investigadora & 3,23 & 1,38 \\
\hline Dificultades idiomáticas & 3,07 & 1,31 \\
\hline Falta de competitividad/presión & 3,01 & 1,32 \\
\hline Falta de facilidades para acometer acciones de movilidad & 2,94 & 1,33 \\
\hline Excesiva rotación en la docencia & 2,76 & 1,39 \\
\hline Juventud del departamento & 2,64 & 1,29 \\
\hline Inexistencia o existencia irregular de cursos de doctorado & 2,58 & 1,38 \\
\hline
\end{tabular}

Tabla VII. Opiniones sobre la productividad a nivel individual

\begin{tabular}{|c|c|c|}
\hline Causa: & Media & DT \\
\hline Tiempo dedicado a la docencia & 3,57 & 1,34 \\
\hline Esfuerzo no recompensado & 3,54 & 1,41 \\
\hline Falta de formación en metodología de investigación & 3,43 & 1,36 \\
\hline Falta de reconocimiento de la investigación en la asignación de docencia & 3,33 & 1,36 \\
\hline Desmotivación por rechazos (proyectos, sexenios, acreditaciones) & 3,28 & 1,57 \\
\hline Tiempo dedicado a la gestión universitaria & 3,20 & 1,36 \\
\hline Dificultades para la formación de grupos de investigación & 3,15 & 1,47 \\
\hline Escasa tradición investigadora del departamento & 3,15 & 1,52 \\
\hline Escasez de revistas específicas de calidad en Contabilidad & 3,13 & 1,36 \\
\hline Falta de financiación & 3,13 & 1,31 \\
\hline Dificultades personales para acometer acciones de movilidad & 3,11 & 1,40 \\
\hline Dificultades idiomáticas & 2,91 & 1,36 \\
\hline Alta rotación en la docencia & 2,65 & 1,38 \\
\hline Inexistencia o existencia irregular de cursos de doctorado & 2,64 & 1,34 \\
\hline Tiempo dedicado al ejercicio profesional & 1,97 & 1,28 \\
\hline
\end{tabular}


Si comparamos los tres principales motivos que, de acuerdo con los encuestados originan diferencias de productividad, observamos que el profesor en Contabilidad considera la desmotivación por rechazos como una de las principales causas que justifican la menor productividad de sus compañeros, tanto a nivel de área como de departamento. Sin embargo, a nivel individual prevalecen otras como el tiempo dedicado a la docencia, el esfuerzo no recompensado o la falta de formación en metodología de investigación. Si analizamos si existen diferencias significativas entre las causas que originan diferencias de productividad en los niveles comentados, encontramos que en relación a falta la formación metodológica no existen diferencias significativas. No obstante, la desmotivación por rechazos parece ser un ítem con diferencias de opinión sobre sus efectos en productividad a nivel de área (media 3,91), departamento $(3,86)$ e individual $(3,28)$, siendo las diferencias estadísticamente significativas.

\section{FACTORES DETERMINANTES DE LAS DIFERENCIAS DE OPINIÓN SOBRE LA PRODUCCIÓN CIENTÍFICA}

En línea con las hipótesis establecidas, hemos analizado si existen diferencias de opinión por edades, tamaño de universidad, categoría profesional o sexenios para cada uno de los niveles analizados en cuanto a productividad: área, departamento e individual. A continuación pasamos a comentar las que han resultado más significativas.

En primer lugar, observamos diferencias significativas por grupos de edad en relación a las con- secuencias de la calidad de los cursos de doctorado (Tabla VIII). Así, el grupo más joven (menor o igual a 30 años) no cree, por término medio, que la mayor o menor calidad de los cursos de doctorado influya sobre la productividad del área, mientras que el profesorado de mayor edad mantiene una posición más neutral o favorable hacia esta causa. Por otro lado, el profesorado de universidades pequeñas atribuye más las diferencias de productividad por área a la falta de formación en metodología de la investigación que el de universidades de mayor tamaño. La categoría profesional no parece influir en la opinión sobre la productividad a nivel de área, por lo que para ese ámbito aceptaríamos sólo las hipótesis $\mathrm{H} 1$ y $\mathrm{H} 2$.

Llama la atención que las diferencias a nivel departamental sólo sean significativas cuando analizamos la existencia o no de tramos de investigación, aceptando la hipótesis H4. Apreciamos que los profesores que cuentan con algún tramo de investigación son más críticos con las causas que motivan la mayor o menor productividad de su departamento, considerando que se debe, fundamentalmente, a la presencia de actitudes acomodadas y atribuyendo menor peso que el grupo de profesores sin sexenio a otras causas como las dificultades de formación o la inexistencia o existencia irregular de cursos de doctorado (Tabla IX). Respecto a las causas de las diferencias de productividad a nivel de departamento no existen diferencias estadísticamente significativas ni por edad, ni por categoría profesional ni por el tamaño de la universidad, por lo que se rechazan para ese nivel las hipótesis $\mathrm{H} 1, \mathrm{H} 2$ y $\mathrm{H} 3$.

Tabla VIII. Análisis de las diferencias de productividad a nivel de área según tamaño de la universidad y edad de los encuestados

\begin{tabular}{lccc}
\hline Diferencias productividad área & Media & Chi-2 & Signif \\
\hline Falta de formación metodológica & & & \\
\hline Universidades pequeñas & 3,9 & 10,502 & 0,005 \\
\hline Universidades grandes & 3,13 & & \\
\hline Universidades muy grandes & 3,48 & & \\
\hline Calidad de los cursos de doctorado & & & \\
\hline Menos 30 años & 3,06 & \\
\hline Entre 30 y 45 años & 3,34 & & \\
\hline Más de 45 años & & & \\
\hline
\end{tabular}

Tabla IX. Análisis de diferencias de productividad a nivel de departamento según la posesión de tramos de investigación

\begin{tabular}{|c|c|c|c|}
\hline Diferencias productividad departamento & Media & U-MW & Signif \\
\hline \multicolumn{4}{|l|}{ Dificultades formación grupos investigación } \\
\hline Sexenio & 2,89 & 2693 & 0,001 \\
\hline No sexenio & 3,41 & & \\
\hline \multicolumn{4}{|l|}{ Presencia actitudes acomodadas } \\
\hline Sexenio & 4,11 & 2437 & 0,011 \\
\hline No sexenio & 3,58 & & \\
\hline \multicolumn{4}{|l|}{ Inexistencia cursos doctorado } \\
\hline Sexenio & 2,14 & 2153,5 & 0,005 \\
\hline No sexenio & 2,77 & & \\
\hline
\end{tabular}


En relación a las posibles diferencias en las opiniones sobre la productividad científica a nivel individual (Tabla X), el grupo más joven considera que la desmotivación por rechazos es la mayor causa de diferencias en productividad para el profesorado universitario en Contabilidad, no otorgando el resto de grupos de edad puntuaciones tan altas a este ítem.

Por tamaño de universidades, la falta de formación en metodologías de investigación vuelve a aparecer como causa de diferencias de productividad y de nuevo son los profesores de las universidades pequeñas los que dan más importancia a este ítem. Por otro lado, el profesorado de universidades muy grandes da más importancia a la escasa tradición investigadora del departamento como causa de la diferencia de productividad a nivel individual que el profesorado de universidades de menor tamaño. Por categorías de profesorado, el personal incluido dentro de la categoría de Otros y el Laboral No Doctor es quien más atribuye a las dificultades personales para la movilidad las causas de su menor productividad. De nuevo el profesorado con tramos de investigación resulta ser más crítico en cuanto a las causas que motivan las diferencias de productividad individual, atribuyendo menor peso a razones de docencia (alta rotación y tiempo dedicado) que el grupo que no cuenta con ningún tramo de investigación.

En consecuencia, tanto la edad, como el tamaño de la universidad, el poseer o no tramos de investigación y la categoría profesional, influyen en la opinión sobre las diferencias de productividad de los profesores universitarios de Contabilidad a nivel individual, aceptándose las hipótesis $\mathrm{H} 1, \mathrm{H} 2$, H3 y H4 para este análisis.

\section{CONSIDERACIONES FINALES}

Este trabajo se realiza basándose en los resultados de una encuesta dirigida a los 910 miembros de ASEPUC para conocer, entre otras cuestiones, sus opiniones sobre las posibles causas que pueden explicar la diferencia entre la producción científica en Contabilidad y en otras áreas. Asimismo, hemos analizado las diferencias de opinión por edades, tamaño de universidad, categoría profesional o sexenios para cada uno de los niveles de productividad analizados.

Tabla X. Análisis de las diferencias de productividad a nivel individual según edad, tamaño de universidad, categoría profesional y posesión de tramos de investigación

\begin{tabular}{|c|c|c|c|}
\hline Diferencias productividad individual & Media & Estadísticos & Signif \\
\hline \multicolumn{4}{|l|}{ Desmotivación por rechazos } \\
\hline Menos 30 años & 4,17 & 5,600 & 0,06 \\
\hline Entre 30 y 45 años & 3,08 & & \\
\hline Más de 45 años & 3,6 & & \\
\hline \multicolumn{4}{|l|}{ Falta formación metodología } \\
\hline Universidades pequeñas & 3,72 & 9,237 & 0,01 \\
\hline Universidades grandes & 2,87 & & \\
\hline Universidades muy grandes & 3,5 & & \\
\hline \multicolumn{4}{|c|}{ Escasa tradición investigadora departamento } \\
\hline Universidades pequeñas & 2,55 & 9,307 & 0,01 \\
\hline Universidades grandes & 3,26 & & \\
\hline Universidades muy grandes & 3,44 & & \\
\hline \multicolumn{4}{|l|}{ Dificultades personales para movilidad } \\
\hline Funcionario doctor & 3,06 & 9,279 & 0,054 \\
\hline Funcionario no doctor & 2,78 & & \\
\hline Laboral doctor & 2,63 & & \\
\hline Laboral no doctor & 3,32 & & \\
\hline Otros & 3,89 & & \\
\hline \multicolumn{4}{|l|}{ Alta rotación docencia } \\
\hline Sexenio & 2,18 & 1928 & 0,003 \\
\hline No sexenio & 2,86 & & \\
\hline \multicolumn{4}{|l|}{ Tiempo dedicado docencia } \\
\hline Sexenio & 2,67 & 1475,5 & 0,000 \\
\hline No sexenio & 3,95 & & \\
\hline
\end{tabular}


A pesar de que los resultados muestran un incremento del tiempo dedicado a tareas de investigación, existe una opinión claramente mayoritaria acerca de que la producción científica en Contabilidad es menor que en otras áreas. Para explicar las causas de esta situación, los profesores que han participado en la encuesta se decantan a nivel global por la desigualdad de criterios con respecto a otras áreas y la dificultad para diferenciar entre la investigación y los trabajos orientados a la mejora de la práctica profesional. Ahora bien, existen diferencias por edad y tamaño de universidad, ya que el grupo más joven no cree, por término medio, que la mayor o menor calidad de los cursos de doctorado influya en la productividad del área, mientras que el profesorado de universidades pequeñas atribuye más las diferencias de productividad por área a la falta de formación en metodología de investigación, respecto al profesorado de universidades de mayor tamaño.

En cuanto a las causas de las diferencias de productividad a nivel de cada universidad o departamento, sobresalen la desmotivación por los rechazos sufridos y la presencia de actitudes acomodadas, seguidas por la falta de incentivos a la investigación, tanto en forma de financiación, como de reconocimiento al asignar la docencia. En este ámbito no se aprecian diferencias de opinión por edad, tamaño de universidad o categoría profesional. Sin embargo, las diferencias a nivel departamental son significativas cuando analizamos la existencia o no de tramos de investigación. Los profesores universitarios de Contabilidad que cuentan con algún tramo de investigación son más críticos con las causas que motivan la mayor o menor productividad de su departamento, considerando que se debe fundamentalmente a la presencia de actitudes acomodadas y atribuyendo menor peso que el grupo de profesores sin sexenio a otras causas como las dificultades de formación o la inexistencia o existencia irregular de cursos de doctorado.

En el plano individual resaltan como causas explicativas el tiempo dedicado a la docencia, el esfuerzo no recompensado y la falta de formación metodológica. Curiosamente, la que se señala como menos influyente es el tiempo dedicado al ejercicio profesional, lo que puede explicarse porque presumiblemente quienes más se dedican a ésta sean los que menos hayan respondido la encuesta. A nivel individual apreciamos importantes diferencias por grupos. Así, el grupo más joven considera que la causa más importante de las diferencias de productividad del profesorado universitario de Contabilidad se debe a la desmotivación por rechazos. Por tamaño de universidades, la falta de formación en metodologías de investigación vuelve a aparecer como causa de diferencias de productividad y de nuevo son las universidades pequeñas las que dan más importancia a este ítem. Por otro lado, el profesorado de universidades muy grandes da más importancia a la escasa tradición investigadora del departamento como causa de diferencia de productividad a nivel individual, siendo el personal incluido dentro de las categorías de Otros y de Laboral No Doctor el que más atribuye a dificultades personales para la movilidad las causas de su menor productividad. Por su parte, el profesorado con sexenios atribuye menor peso a razones de docencia (alta rotación y tiempo dedicado) que el grupo que no cuenta con tramos para explicar las productividades a nivel individual.

Por tanto, debemos incidir en la importancia de la edad como factor condicionante no sólo de la mayor o menor productividad del profesor, sino también de la opinión sobre la misma dentro del área. Así, es el profesorado más joven, probablemente por necesidad, el que parece haberse adaptado mejor al "cambio en las reglas del juego", aunque es preciso señalar como riesgos una menor dedicación a la docencia y una tendencia de la investigación hacia temas "más fácilmente publicables" en revistas de impacto.

Por último, hemos de señalar que aunque los resultados obtenidos están lógicamente influenciados por la muestra, ésta representa bastante fielmente la distribución de la población por categorías profesionales. No obstante, los datos relativos al porcentaje de doctores, a los tiempos dedicados a la investigación, así como la participación en proyectos de investigación competitivos, entre otros, ponen de manifiesto que de existir un sesgo en la muestra, éste podría derivarse de que los que han respondido pertenecen a un grupo de profesores en el que existe una inquietud y una preocupación importante por la investigación. A nuestro entender, este posible sesgo más que limitar las posibles inferencias que se pudieran derivar de esta parte del estudio centrada en las posibles causas de la menor productividad científica en Contabilidad, por el contrario, las harían más relevantes y significativas.

\section{NOTAS}

[1] En concreto, Illueca y García (2009) confirmaron que el profesorado de los departamentos de Contabilidad de las universidades públicas españolas se distribuía de la siguiente manera: 7\% Catedráticos de Universidad, 24\% Titulares de Universidad, 20\% Titulares de Escuela Universitaria, 9\% Contratados Doctores, 4\% Ayudantes y 36\% Asociados y Colaboradores.

\section{BIBLIOGRAFÍA}

Alexander, J., Lecoutre, M. y R. Scherer. (2007). A global comparison of business journal ranking systems. Journal of Education for Business, July/ August: 321-327. http://dx.doi.org/10.3200/ JOEB. 82.6.321-328

Alonso-Arroyo, A.; Bolaños-Pizarro, M.; González-Alcaide, G.; Villamón, M. y R. Aleixandre-Benavent. (2010). Análisis de género, productividad científica y colaboración de las profesoras universitarias de Ciencias de la Salud en la Comunidad Valen- 
ciana (2003-2007). Revista Española de Documentación Científica, 33 (4): 624-642. http://dx.doi. org/10.3989/redc.2010.4.764

Alonso-Arroyo, A.; Pulgarín, A. e I. Gil-Leiva. (2006). Análisis bibliométrico de la producción científica de la Universidad Politécnica de Valencia 1973-2001. Revista Española de Documentación Científica, 29 (3): 345-363.

Amat, O.; Blake, J.; Gowthorpe, C. y E. Oliveras. (1998). Análisis de autores, citas y revistas de Contabilidad en España. Revista Española de Financiación y Contabilidad, XXVII (96): 821-850.

Amat, O.; Oliveras, E. y J. Blake. (2001). Revista Española de Financiación y Contabilidad (1985-1999): un análisis retrospectivo. Revista Española de Financiación y Contabilidad, XXX (108): 529-552.

Argilés, J. (2009). Investigación y carrera académica en Contabilidad: referencia específica a España, III Congrés Català de Comptabilitat i Direcció, Barcelona.

Argilés, J. y J. García (2011). Accounting Research: a Critical View of the Present Situation and Prospects. Revista de Contabilidad-Spanish Accounting Review, 14 (2): 9-34. http://dx.doi.org/10.1016/ S1138-4891(11)70026-7

Astin, H.S. y D. Davis. (1985). Research Productivity Across the Life and Career Cycles: Facilitators and Barriers for Women. In M. F. Fox, cd., Scholarly Wrilirrg and Publishing: Issues, Problems and Solutions. Boulder, Colo.: Westview Press.

Bayer, A.E., y J. Dutton. (1977). Career age and research-professional activities of academic scientists. Journal of Higher Education, 48: 259-282. http://dx.doi.org/10.2307/1978680

Bayo, A. y M. Larraza. (2012). La investigación en recursos humanos en España de 2001 a 2010: ¿la década prodigiosa? Cuadernos de Economía y Dirección de la Empresa, 15, 181-191. http://dx.doi. org/10.1016/j.cede.2012.04.006

Bellas, M.L. y R.K. Toutkoushian. (1999). Faculty time allocations and research productivity". The $R e$ view of Higher Education, 22 (4): 367-390. http:// dx.doi.org/10.1353/rhe.1999.0014

Benítez, N. y C.G. Benavides. (2012). Investigación en innovación tecnológica: un estudio bibliométrico de Revista Europea de Dirección y Economía de la Empresa. Revista Europea de Dirección y Economía de la Empresa, 21, 157-168. http://dx.doi. org/10.1016/S1019-6838(12)70003-3

Bentley, R.J., y R.T. Blackburn. (1990). Relationship of faculty publication performance with age, career age, and rank, Paper presented at the annual meeting of the Association for the Study of Higher Education, Portland, OR.

Bland, C.J.; Finstad, D.A. y J.G. Staples. (2005). A Theoretical, Practical, Predictive Model of Faculty and Departament Research Productivity. Academic Medicine, 8 (3): 225-237. http://dx.doi. org/10.1097/00001888-200503000-00006

Bolaños-Pizarro, M.; Vidal-Infer, A.; Navarro-Molina, C. ; Valderrama-Zurián, J.C.; González-Alcai- de, G. y R. Aleixandre-Benavent. (2009). Análisis de la productividad científica y visibilidad de las publicaciones sobre usabilidad (1971-2005). Revista Española de Documentación Científica, 32 (1): 9-21.

Bracho-López, R.; Maz-Machado, A.; Gutiérrez-Arenas, P.; Torralbo-Rodríguez, M.; Jiménez-Fanjul, N.N. y N. Adamuz-Povedano. (2012). La investigación en Educación Matemática a través de las publicaciones científicas españolas. Revista Española de Documentación Científica, 35 (2): 262-280. http:// dx.doi.org/10.3989/redc.2012.2.870

Brown, L.D. (1996). Influential accounting articles, individuals, Ph.D. granting institutions and faculties: a citational analysis. Accounting, Organizations and Society, 21(7/8): 723-54. http://dx.doi. org/10.1016/0361-3682(96)00012-8

Brusca, I. (2010). Treinta años de investigación en Contabilidad y Gestión Pública en España. Revista de Contabilidad-Spanish Accounting Review, 13 (2): 175-209. http://dx.doi.org/10.1016/S11384891(10)70016-9

Bucheit, S., Collins, D. y A. Reitenga. (2002). A crossdiscipline comparison of top-tier academic publication rates: 1997-1999. Journal of Accounting Education, 20: 123-130. http://dx.doi.org/10.1016/ S0748-5751(02)00003-9

Cañibano, L. y J.A. Gonzalo (1997). Los Programas de Investigación en Contabilidad. Revista de Contabilidad-Spanish Accounting Review, 0 (0): 57-95.

Carmona, S.; Gutiérrez, I. y M. Cámara. (1999). A profile of European accounting research: evidence from leading research journals. European Accounting Review, 8 (3): 463-480. http://dx.doi. org/10.1080/096381899335880

Casanueva, C.; Escobar, B. y C. Larrinaga. (2007). Red social de Contabilidad en España a partir de los tribunales de tesis. Revista Española de Financiación y Contabilidad, XXXVII (136): 703-724.

Casanueva, C. y F. Caro (2013). Spanish communication academia: scientific productivity versus social activity. Comunicar, 41: 61-70. http://dx.doi. org/10.3916/C41-2013-06

Cavero, J.A. y V. Ferrández. (2013). El sexenio de investigación en Contabilidad, Educade, 4: 5-21.

Codina-Canet, M.; Olmeda-Gómez, C. y A. PerianesRodríguez. (2013). Análisis de la producción científica y de la especialización temática de la Universidad Politécnica de Valencia. Scopus (20032010). Revista Española de Documentación Científica, 36 (3). http://dx.doi.org/10.3989/ redc. 2013.3.942

Correa Domènech, M.; García Blandón, J. y R. Úbeda Sales. (2009). Análisis de la producción científica en Economía Financiera: 1995-2006. Revista Española de Documentación Científica, 32 (2): 83-104. http://dx.doi.org/10.3989/redc.2009.2.671

De las Heras, G. y G. Polo. (2009). La evaluación de la investigación universitaria en ciencias jurídicas en el marco general español. Revista de Educación Superior, XXXVIII (2): 63-79. 
Donoso, R. (2005). Estado actual de la investigación en Historia de la Contabilidad. Revista de Contabilidad-Spanish Accounting Review, 15: 19-44.

Dundar, H. y D.R. Lewis. (1998). Determinants of Research Productivity in Higher Education. Research in Higher Eduaction, 39 (6): 607-631. http:// dx.doi.org/10.1023/A:1018705823763

Escobar, B., Lobo, A. y C. Rocha. (2005). La Investigación Empírica en Contabilidad de Gestión en España: Análisis de las Publicaciones Españolas. Revista Española de Financiación y Contabilidad, 124: $183-210$

Fogarty, T.J. (2004). Sustained research productivity in accounting. Global perspectives on accounting education, 1: 31-58.

Fogarty, T.J. y J.M. Ruhl. (1997). Institutional antecedents of accounting faculty research productivity: a LISREL study of the "Best and Brightest". Issues in Accounting Education, 12: 27-48.

Fundación Española para la Ciencia y la Tecnología. (2007). Indicadores bibliométricos de la actividad científica española (1990-2005). Madrid: FECYT

García, M.C.; Gonzalo, J.A.; Jiménez, J.A. y C. TaraviIlo. (1986). Análisis de autores y citas en revistas contables españolas. Técnica Contable, 353-366.

García, M.C. (2007). Institutional and Individual Factors Associated with Faculty Scholarly Productivity. Dissertation Abstracts International (AAT 3276618).

Guardiola-Víllora, A. y L. Basset-Salom. (2012). Análisis de la actividad investigadora en arquitectura y urbanismo según los resultados de la CNEAI. Revista Española de Documentación Científica, 35 (3): 393-413. http://dx.doi.org/10.3989/ redc. 2012.3.909

Hernández, E. (2005). Un lustro de investigación histórico-contable en España (2001-2005). Revista de Contabilidad-Spanish Accounting Review, 8 (15), 45-94.

Ibañez, A.; Bielza, C. y P. Larrañaga. (2013). Análisis de la actividad científica de las universidades públicas españolas en el área de las tecnologías informáticas. Revista Española de Documentación Científica, 36 (1) e002. doi: http://dx.doi.org/10.3989/ redc. 2013.1.912

Illueca, M. ; Garcia, M.A. (2009). "Evolucion y situación actual de la investigación contable en España". Ponencia presentada en las Jornadas de debate y reflexión sobre la situación actual y perspectivas en la investigación contable en España. ASEPUC-Universidad de Salamanca.

Jiménez, E.; Moya, F. y E. Delgado. (2003). The evolution of research activity in Spain: The impact of the National Comission for the Evaluation of Research Activity (CNEAI). Research Policy, 32 (1): 123-142. http://dx.doi.org/10.1016/S0048-7333(02)00008-2

Jiménez-Contreras, E.; Robinson-García, N. y A. Cabezas-Clavijo. (2011). Productivity and impact of Spanish researchers: reference thresholds within scientific areas. Revista Española de Documentación Científica, 34 (4): 505-526. http://dx.doi. org/10.3989/redc.2011.4.828
Jones, M. y R. Roberts. (2005). International publishing patterns: an investigation of leading UK and US accounting and finance journals. Journal of Business Finance and Accounting, 32(5/6): 1107-1140. http://dx.doi.org/10.1111/j.0306686X.2005.00624.X

Khalifa, R. y P. Quattrone -Eds.- (2008). Accounting and academia: career systems, networks and what matters. European Accounting Review, Special Section, 17 (1): 65-191.

Kortrlick, J.E.; Bertlett, I.C. y H.A.W. Higgings. (2002). Factors associated with research productivity of agricultural education faculty. Journal of Agricultural Education, 43 (2): 1-40.

Larrán-Jorge, M.; Escobar-Pérez, B. y E. García-Meca. (2013). El sistema de acreditación nacional: la opinión de los profesores universitarios de Contabilidad. Revista Española de Documentación Científica, 36(3):e015. doi: http://dx.doi.org/10.3989/ redc. 2013.3.947

Larrinaga, C. (2005). Producción científica en Contabilidad: el caso de España (1992-2002). Revista Española de Financiación y Contabilidad, XXXIV (125): 363-394.

Lee, T. (1997). The editorial gatekeepers of the accounting academy. Accounting, Auditing and Accountability Journal, 10 (1): 11-30. http://dx.doi. org/10.1108/09513579710158694

López-Berna, S.; Papí-Gálvez, N. y M. Martín-Llaguno. (2011). Productividad científica en España sobre las profesiones de comunicación entre 1971 y 2009. Revista Española de Documentación Científica, 34 (2): 212-231. http://dx.doi.org/10.3989/ redc. 2011.2 .801

Lunkes, R.J.; Ripoll, V.M. y F. Silva. (2011). Contabilidad de Gestión: un estudio en revistas de Brasil, España y de lengua española. Revista de Contabilidade e Organizações, 5 (13): 132-150. http:// dx.doi.org/10.11606/rco.v5i13.34808

Maranto, C. y C. Streuly. (1994). The determinants of accounting professors publishing productivity-the early career. Contemporary Accounting Research, 10, 2: 387-407. http://dx.doi. org/10.1111/j.1911-3846.1994.tb00399.x

Marcos Recio, J.C.; Martínez Pestaña, P. y M.F. Blasco López. (2012). Producción y dirección de tesis doctorales sobre publicidad en la universidad española (1971-2010). Revista Española de Documentación Científica, 35 (3): 433-452. http://dx.doi. org/10.3989/redc.2012.3.890

Mathieu, R. y B. McConomy. (2003). Productivity in "top-ten" academic accounting journals by researchers at Canadian universities. Canadian Accounting Perspectives, 2(1): 43-76. http://dx.doi. org/10.1506/J5G6-2WXL-H8M1-YL92

Maz-Machado, A.; Torralbo-Rodríguez, M.; VallejoRuiz, M. y R. Bracho-López. (2010). Análisis bibliométrico de la producción científica de la Universidad de Málaga en el Social Sciences Citation Index (1998-2007). Revista Española de Documen- 
tación Científica, 33 (4): 582-599. http://dx.doi. org/10.3989/redc.2010.4.757

Moya, S. y D. Prior. (2008). ¿Quién publica en las revistas españolas de Contabilidad? Análisis bibliométrico 1996-2005. Revista Española de Financiación y Contabilidad, XXXVII (138): 1-22.

Panozzo, F. (1997). The making of the good academic accountant. Accounting, Organizations and Society, 22 (5): 447-480. http://dx.doi.org/10.1016/ S0361-3682(96)00043-8

Pastor-Ruiz, F.; Torrado-Morales, S.; Gil-Felipe, J. y N. Lorenzo-Escolar. (2013). Análisis de las publicaciones del personal docente e investigador de las facultades españolas de Bellas Artes, Revista Española de Documentación Científica, 36 (3), doi: http://dx.doi.org/10.3989/redc.2013.3.961

Raffournier, B. y A. Schatt. (2009). Is European accounting research fairly reflected in European journals? Working paper.

Richart-Ramón, A.; Martínez-Blasco, M. y J. GarcíaBlandón. (2011). Análisis de la producción científica sobre Gobierno Corporativo a través del ISI Web of Science. Revista Española de Documentación Científica, 34 (1): 79-101. http://dx.doi.org/10.3989/ redc. 2011.1 .776

Rojas-Sola, J.I.; Navarrete-Cortés, J.; FernándezLópez, J.A. y J.A. Chaichio-Moreno. (2008). Producción científica del área de expresión gráfica en la ingeniería en las universidades españolas: una aproximación a la base de datos ISI. Revista Española de Documentación Científica, 31 (2): 190-204.

Ronda, G.A. y L.A. Guerras. (2013). Red de cooperación institucional de investigación en dirección de empresas en España en torno a la revista CEDE: 1998-2010. Cuadernos de Economía y Dirección de la Empresa, 16, 1-16. http://dx.doi.org/10.1016/j. cede.2012.06.003

Santos, G. (2012). Aproximaciones a un análisis bibliométrico de la Revista Internacional Legis de Contabilidad \& Auditoría 2000-2012. Revista Internacional Legis de Contabilidad \& Auditoría, 50, 183-226.

Sanz, L. (1995). Research actors and the state: research evaluation and evaluation of science and technology policies in Spain. Research Evaluation, 5 (1): 79-88.

Torres-Salinas, D.; Muñoz-Muñoz, A. y E. JiménezContreras. (2011). Análisis bibliométrico de la situación de las mujeres investigadoras de Ciencias Sociales y Jurídicas en España. Revista Española de Documentación Científica, 34 (1): 11-28. http:// dx.doi.org/10.3989/redc.2011.1.794

Zeff, S. (1996). A Study of Academic Research Journals in Accounting, Accounting Horizons, 10 (3): 158-177. 